\title{
Relationship of lunar phases and sex of the foetus: a retrospective study
}

\author{
Aman Deep Raj ${ }^{1 *}$, Roshni Abichandani ${ }^{2}$, Harish Sethi ${ }^{3}$
}

\begin{abstract}
${ }^{1}$ Department of Obstetrics and Gynaecology, Military Hospital, Bathinda, Punjab, India ${ }^{2}$ Department of Obstetrics and Gynaecology, Military Hospital, Jaipur, Rajasthan, India ${ }^{3}$ Department of Paediatrics Military Hospital Bathinda, Punjab, India
\end{abstract}

Received: 25 May 2021

Revised: 21 June 2021

Accepted: 22 June 2021

\author{
*Correspondence: \\ Dr. Aman Deep Raj, \\ E-mail: amandeepraj@yahoo.co.uk
}

Copyright: (c) the author(s), publisher and licensee Medip Academy. This is an open-access article distributed under the terms of the Creative Commons Attribution Non-Commercial License, which permits unrestricted non-commercial use, distribution, and reproduction in any medium, provided the original work is properly cited.

\begin{abstract}
Background: The relation of lunar phases at the time of conception to the sex of the child at birth has been touted as a method of sex selection by various birth calendars with controversial results. It is made to believe that indeed there is a relation of lunar phase at the time of conception/intercourse which results in birth of a particular gender. The purpose of this study was to examine the influence of the lunar phase on the possible relationship between lunar position at EDD/LMP and the gender of the child.

Methods: One thousand and five deliveries were retrospectively analysed from 1 January 2016 to 31 December 2018 at the department of obstetrics and gynaecology of military hospital, Gwalior (latitude and longitude coordinates: 26.218287, 78.182831). Females having regular menstrual periods history around conception and in general their periods have been normal and regular were included in the study. Females having irregular menstrual periods history or having oligomenorrhoea/polymenorrhoea, ART/IVF conceptions were excluded from the study. Exact lunar phases were determined at the time of EDD and not with their LMP since that would automatically corelate well with LMP because of regularity of their periods. The actual date of birth was not considered while corelating with moon phase because of obvious reason of unexpected timing of labour.

Results: The analysis revealed no significant correlation of gender of the child to lunar phases at EDD. This would indirectly corelate well with the LMP since the inclusion criterion had females having normal and regular menstrual periods.

Conclusions: There is no predictable influence of the lunar phase on the gender of the baby. As expected, and in agreement with some recent studies this pervasive myth is not evidence based.
\end{abstract}

Keywords: Lunar cycle, Lunar phase, Moon phase, Sex of baby, Sex selection, Gwalior, Birth calenders

\section{INTRODUCTION}

The lunar cycle has long been suspected to influence biological phenomena. Early myths associate the moon with growth efficiency of crops, animal reproduction and behaviour and human fertility. The ancient Greeks worshiped Artemis as the goddess of the moon, childbirth and fertility. ${ }^{1}$ There are many anecdotes about diets and sexual positions that help people conceive a child of the desired sex. Some people believe that the position of the moon at the moment of conception determines the sex of the foetus, a positive sign indicates the child will be a boy and a negative sign indicates a girl. The Chinese birth chart is based on this theory and is related closely to lunar cycles. The chart predicts the sex of a child based on the mother's age and the month of its conception. ${ }^{2}$

The lunar phase or moon phase is the shape of the moon's directly sunlit portion as viewed from Earth. The phases of the moon, as we know them, correspond to the synodic 
cycle, the ends and beginnings of each lunar month are dictated by a visible full moon and each month can be easily divided into quarters or lunar phases. ${ }^{3}$ There are four principal lunar phases, the new moon, first quarter, full moon and last quarter (also known as third or final quarter), when the moon's ecliptic longitude is at an angle to the sun (as viewed from Earth) of $0^{\circ}, 90^{\circ}, 180^{\circ}$ and $270^{\circ}$, respectively. ${ }^{4}$ Each of these phases appears at slightly different times at different locations on Earth. During the intervals between principal phases are intermediate phases, during which the moon's apparent shape is either crescent or gibbous. On average, the intermediate phases last one-quarter of a synodic month, or 7.38 days. ${ }^{4}$ The descriptor waxing is used for an intermediate phase when the moon's apparent shape is thickening from new to a full moon and waning when the shape is thinning. The longest duration between full moon to new moon (or new moon to full moon) lasts about 15 days and 14.5 hours, while the shortest duration between full moon to new moon (or new moon to full moon) lasts only about 13 days and 22.5 hours. ${ }^{4}$

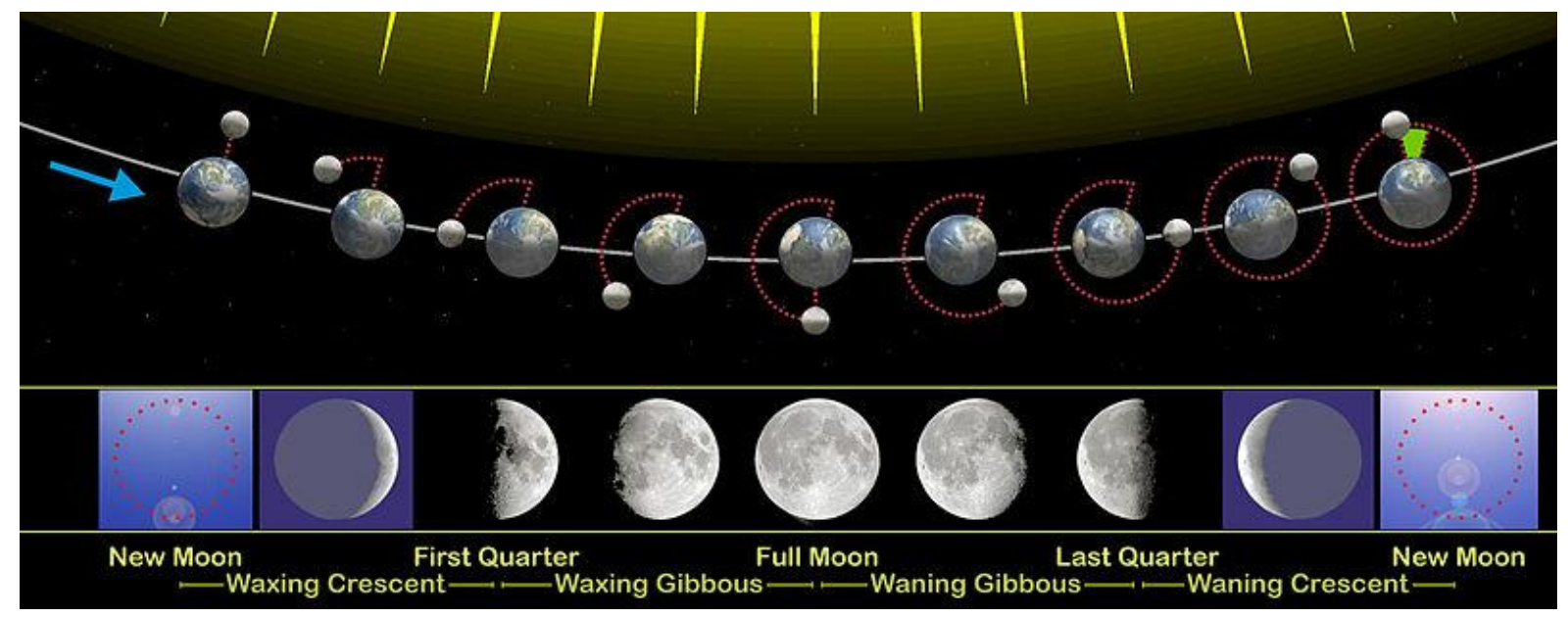

Figure 1: The phases of the moon as viewed looking southward from the northern hemisphere; each phase would be rotated $180^{\circ}$ if seen looking northward from the southern hemisphere; the upper part of the diagram is not to scale as the moon is much farther from Earth than shown here. ${ }^{4}$

\section{Background}

Lunar cycles have important effects on several biological events. Controversy exists about the influence of lunar cycles on offspring sex ratio. In dogs sex offspring distribution was not affected by season, whereas lunar phase had a significant effect $(\mathrm{p}<0.05) .^{5}$

Study done in sheep cattle goats and pigs by Abecia et al showed that in sheep, offspring sex ratio and lunar phase were not correlated, season had a significant $(\mathrm{p}=0.002)$ effect on offspring sex ratio. The proportion of males born of spring and winter mating was significantly higher than it was among offspring born of summer $(p<0.05)$ or autumn $(\mathrm{p}<0.01)$ conceptions. In cattle, moon phase and season did not affect the offspring sex ratio. The overall piglet sex ratio (0.522) and the sex ratios among piglets conceived during a crescent moon and those conceived in summer differed significantly $(\mathrm{p}<0.05)$ from $1: 1 .^{6}$

Effects of full moon and no moon on the birth of male and female offsprings were studied in Indian couples of the age group 20 to 40 years. It was observed that 42 wives who were conceived within 24 hours of ovulation at full moon gave birth of 40 male and 2 female babies. On the other hand, 40 women conceived on the day of ovulation 3 days prior to full moon gave birth of 13 male and 27 female babies. But only 5 women conceived on no moon, all of them gave birth of female babies. It was also observed that vaginal $\mathrm{pH}$ of the ovulated women during full moon was alkaline $(\mathrm{pH} 8.7 \pm 0.4)$ while $\mathrm{pH}$ was weak acidic in women ovulated 3 days prior to full moon and no moon $(\mathrm{pH}$ $6.4 \pm 0.5 ; 6.2 \pm 0.5)$. The basal body temperature (BBT) was increased 0.7 degrees $F$ to 1.3 degrees $F$ during the ovulation period when compared with women during the absence of ovulation. But there is an increase in temperature 0.5 degrees $\mathrm{F}$ more in women ovulated in full moon than no moon. Together, these results indicate that alkaline vaginal fluid medium and more rise of BBT during full moon favour conception of male babies. This method gives the couple more chance of having male child if conception occurs in the day of ovulation in full moon and having female child if conception occurs in no moon. ${ }^{7}$

Villamor and his colleagues reviewed records of 2.8 million Swedish births, between 1973 and 2006, to test the accuracy of the Chinese lunar calendar method. The technique involves converting the mother's age and the month of conception to dates on the Chinese lunar calendar, then plugging those dates into a chart that purportedly predicts the baby's sex. Dr. Eduardo Villamor of the UM school of public health and colleagues in 
Sweden and Boston found that the so-called Chinese lunar calendar method of predicting a baby's sex is no more accurate than flipping a coin. ${ }^{8}$

\section{Objective}

Objective of this study was to determine any corelation of moon phase at the time of conception or LMP with gender of the foetus. This will help people make decisions based on scientific data rather than that of anecdotes and hearsay, thus limiting the superstition and saving unnecessary ordeal that a couple may undergo for selecting a sex of the foetus that one may desire.

\section{METHODS}

Given the lack of past evidence of any influence of the moon on human reproduction, we inferred that any effect, if it existed, would be small. Thus, we selected a data set whose sample size was big enough to detect very small variations.

\section{Type of study}

The study conducted was a single centre retrospective study.

\section{Place of study}

The study was conducted at the military hospital, Gwalior, MP, India.

\section{Period of study}

The human data collected for this study was collected during the author's posting to $\mathrm{MH}$ Gwalior over a threeyear period from January 2016 to December 2018 in a retrospective manner.

\section{Selection criteria}

The mothers chosen for this study had normal and regular menstrual periods preconceptionally and may have had fertilisation outside Gwalior city limits which was not considered while conducting the study.

\section{Exclusion criteria}

Women with irregular menstrual periods and abnormally long and short periods were kept outside the study. Similarly, women who conceived with ART/IVF were also kept out of the study.

\section{Procedure}

A total of one thousand and five (1005) deliveries were retrospectively analysed in terms of age and blood group of mothers and the weight of the new born babies were all collected. The exact moon phases were elucidated both for
EDD and actual date of birth. The moon phase on the EDD was corelated with the gender of the child. Only the six phases of moon viz; waxing, waning, 1st quarter, 3rd quarter, full moon and new moon were taken into account while conducting the study and not the exact days of the lunar cycle. Although the EDD was corelated against the phases of the moon but it is presumed that this moon phase corelation holds good for the LMP and the day of conception since the women enrolled had regular menstrual periods. The actual day of delivery was not taken in to consideration since the women underwent spontaneous labour prior to the EDD or had to be induced for delay in onset of labour beyond 40 weeks of gestation.

SPSS ver.20 was used to interpret data and formulate results.

\section{RESULTS}

The mothers selected for the study came from lower to upper middle class socioeconomic strata of serving and retired wives of Indian army personnel from across the length and breadth of the country. Minimum age of mother at conception was 19 years and maximum was 45 years with a mean age of 26.06 years and SD of 3.680. Minimum weight of the new born was $1.30 \mathrm{kgs}$ and maximum weight was $4.60 \mathrm{kgs}$ with a mean weight of $2.9744 \mathrm{kgs}$ and SD of 0.49684 .

Of the total 1005 deliveries which fulfilled the inclusion criteria there were a total of 555 male $(55.2 \%)$ and 450 $(44.8 \%)$ female children were born during the three year period

Number of males and female children born under different moon phases were plotted and chi squared test performed. It was concluded that there was no significant difference between any particular gender selectively being born on a particular phase of the moon cycle. Results were even strongly insignificant especially on full moon and new moon days. The results were not significant either between the waxing and waning phases of the moon and the 1st or 3rd quarter of the moon phase

There was a preponderance of male gender during both the waxing and waning phases of the moon. There was a total of 252 males as compared to 209 females born during the waxing moon and a total of 244 males and 181 females born during the waning moon phase. There was almost equal number of males and females born on other phases of the moon. Out of the total 1005 deliveries males outnumbered females

\section{Chi square test}

Chi square value $=\mathrm{X}^{2}=2.506$.

Degree of freedom $=\mathrm{df}=5$.

$\mathrm{P}=0.776$. 


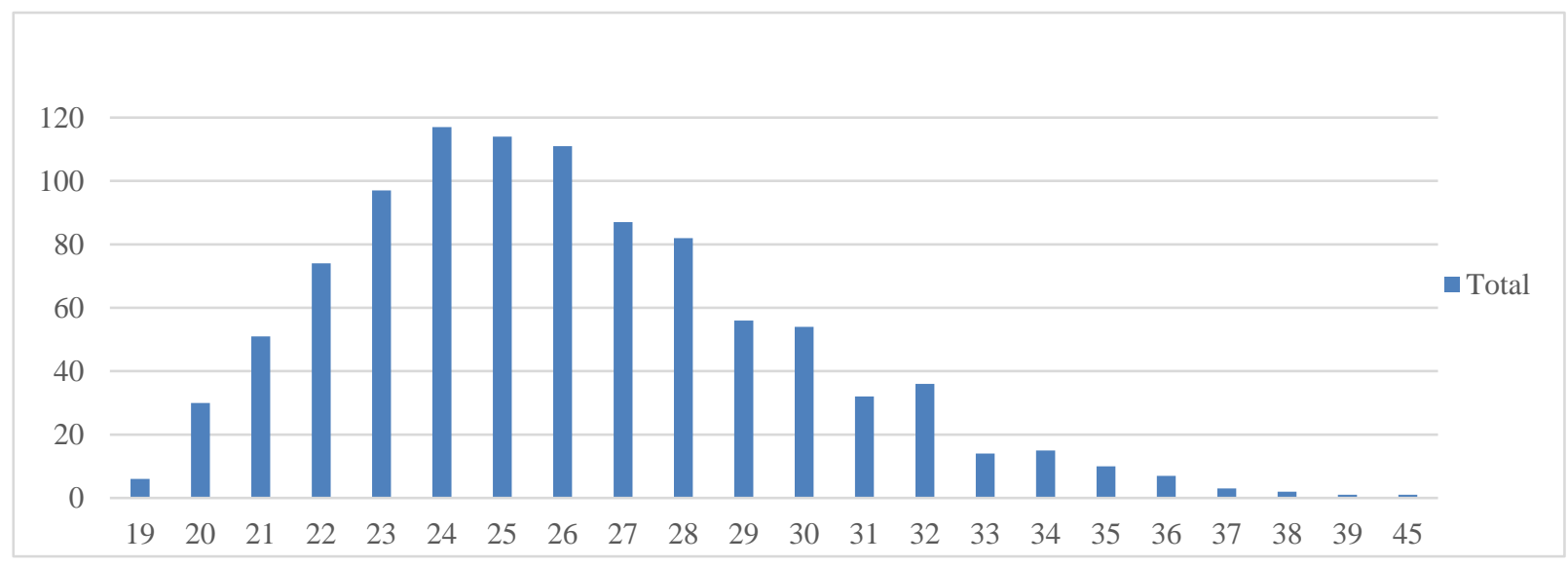

Figure 2: Age distribution of the ladies at the time of delivery; most common age at delivery was 24 years.

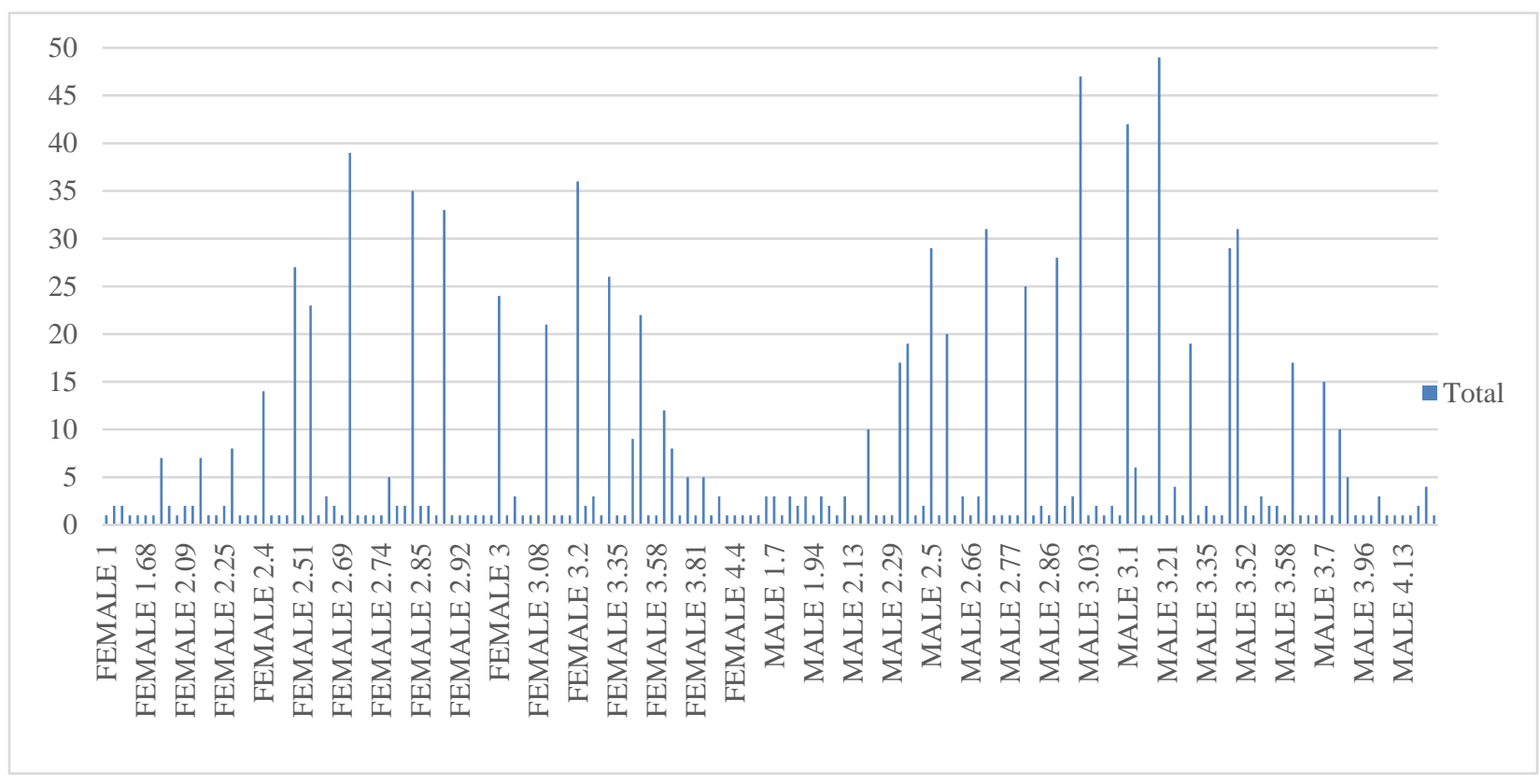

Figure 3: Weight distribution among males and females at the time of delivery; overall male babies were heavier as evident by the chart.

Table 1: Percentage of male and female children.

\begin{tabular}{|lll|}
\hline Sex & Number & Percentage \\
\hline Male & 555 & 55.2 \\
\hline Female & 450 & 44.8 \\
\hline Total & 1005 & 100 \\
\hline
\end{tabular}

Table 2: Gender distribution according to the moon phase.

\begin{tabular}{|llll|}
\hline Moon phase & Female & Male & Total \\
\hline Waxing & 209 & 252 & 461 \\
\hline Waning & 181 & 244 & 425 \\
\hline 3rd quarter & 16 & 15 & 31 \\
\hline New moon & 15 & 16 & 31 \\
\hline Full moon & 15 & 14 & 29 \\
\hline 1st quarter & 14 & 14 & 28 \\
\hline Total & 450 & 555 & 1005 \\
\hline
\end{tabular}




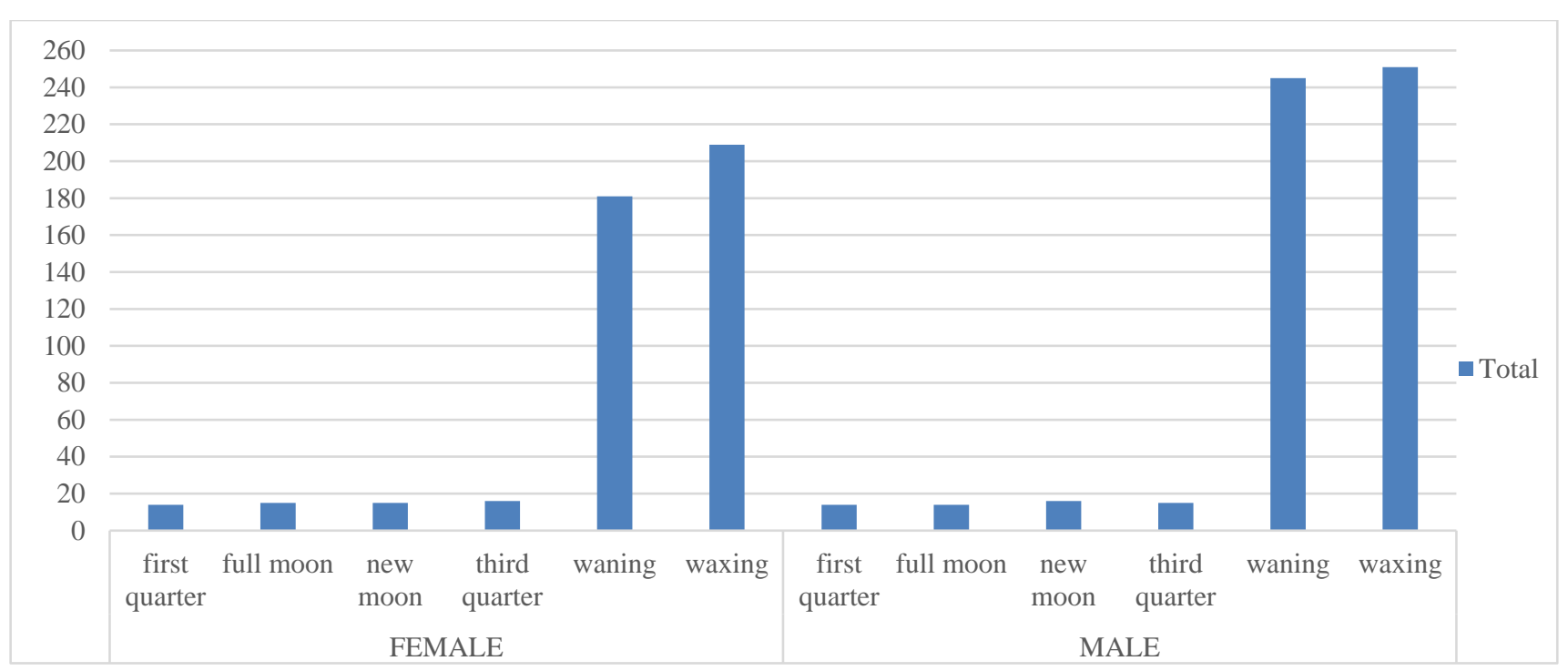

Figure 4: Distribution of total number of male and female sex across the various phases of the moon at the time of EDD.

\section{DISCUSSION}

This study has purely focused on the phases of moon on the day of EDD and the gender of the baby. Strictly speaking this study revealed no significant correlation when a particular moon phase on the EDD is correlated to the sex of the child. These results would corelate directly into no effect of moon phases on the gender of the child as per LMP or the day of conception as there were no irregularities of menstrual periods in the subjects chosen.

Although Sarkar et al (2005) showed that alkaline vaginal fluid medium and more rise of BBT during full moon favour conception of male babies, but, in our study, there appeared no correlation of male sex predominance on conceptions that occurred on full moon days. ${ }^{7}$ In fact, out of the total of 29 births that took place there were 15 females and 14 males at the time of full moon which is statistically insignificant. ${ }^{7}$

Our study is in complete agreement with what Villamor et al has brought out in their study that predicting a baby's sex by Chinese lunar calendar is no more accurate than flipping a coin. ${ }^{8}$

There is a hypothesis that ovum itself may give chemical signal or chemoattractant and select the type of sperm that may fertilize a particular ovum and may result in a particular gender of the foetus. As has been concluded in one of the review articles by Skandhan et al in 2011. ${ }^{9}$

\section{Limitations}

This study can have different results if the exact days of moon phases are plotted against the exact date of conception or ovulation which is very difficult to assess retrospectively and hence beyond the scope of this study. This study simply has given no significant correlation of gender of the child being influenced by the moon phase on EDD. Furthermore, each of the four main phases of moon appears at slightly different times at different locations on Earth. The longest duration between full moon to new moon (or new moon to full moon) lasts about 15 days and 14.5 hours, while the shortest duration between full moon to new moon (or new moon to full moon) lasts only about 13 days and 22.5 hours. So, keeping in view of the above facts there may or may not be any relation of the geographical time and place where the actual conception has taken place.

\section{CONCLUSION}

This study has led to the conclusion that there is no evidence that a particular moon phase determines the sex of the baby a couple is going to have, as far as humans are concerned and the various methods like Chinese calendar of gender selection are nothing but mere anecdotes and has no scientific backing. There may be certain receptors characteristics or chemical composition an ovum might have which can attract a particular type of sperm but that again is beyond the scope of this study. Indeed, there is a growing body of evidence that the ovum does influences the type of sperm which fertilises it. All this requires further study and in future this puzzle of random sex selection by nature may start falling in to line and we may just find the missing blocks which actually fits just in place. All these are corelations worth studying in future but as of now it appears that there is insignificant evidence that the lunar phase has on the selection of gender of the offspring in the humans and it appears purely a random process of what type of sperm meets what type of ova.

Funding: No funding sources Conflict of interest: None declared

Ethical approval: The study was approved by the Institutional Ethics Committee 


\section{REFERENCES}

1. Littleton CS. Mythology: The Illustrated Anthology of World Myth and Storytelling. 1st ed. Duncan Baird Publishers; 2002.

2. Your Chinese Astrology. Fact sheet: How to use chinese baby gender chart? Available at: https://www.yourchineseastrology.com/baby-genderprediction/how-to-use-chinese-birth-chart.htm. Accessed on 10 May 2021.

3. Rotton J, Kelly IW. Much ado about the full moon: a meta-analysis of lunar-lunacy research. Psychol Bull. 1985;97(2):286-306.

4. Wikipedia the free encyclopaedia. Fact sheet: Lunar phase. Available at: https://en.wikipedia.org/ wiki/Lunar_phase. Accessed on 10 May 2021.

5. Alberghina D, Gioè M, Quartuccio M, Liotta L. The influence of lunar cycle at the time of conception on sex offspring distribution in dogs. Chronobiol Int. 2021;1-5.

6. Abecia J, Francisco A, Carlos R. Offspring sex ratio in sheep, cattle, goats and pigs: influence of season and lunar phase at conception. Biol Rhythm Res. 2017;48(3):1-8.

7. Sarkar M Biswas NM. Influence of moonlight on the birth of male and female babies. Nepal Med Coll J. 2005;7(1):62-4.

8. Villamor E, Dekker L, Svensson T, Cnattingius S. Accuracy of the Chinese lunar calendar method to predict a baby's sex: a population-based study. Paed Perinat Epidemiol. 2010;24:398-400.

9. Skandhan KP, Japee J. Hypothesis: who is responsible for sex of offspring? Trend Med Res. 2011;6:161-8.

Cite this article as: Raj AD, Abichandani R, Sethi H. Relationship of lunar phases and sex of the foetus: a retrospective study. Int $\mathrm{J}$ Reprod Contracept Obstet Gynecol 2021;10:3023-8. 